\title{
Differential mtDNA Damage Patterns in a Transgenic Mouse Model of Machado- Joseph Disease (MJD/SCA3)
}

\section{Amanda Ramos, Nadiya Kazachkova,}

Francisca Silva, Patrícia Maciel, Anabela Silva-Fernandes, Sara Duarte-Silva, Cristina Santos, et al.

Journal of Molecular Neuroscience

ISSN 0895-8696

J Mol Neurosci

DOI 10.1007/s12031-014-0360-1

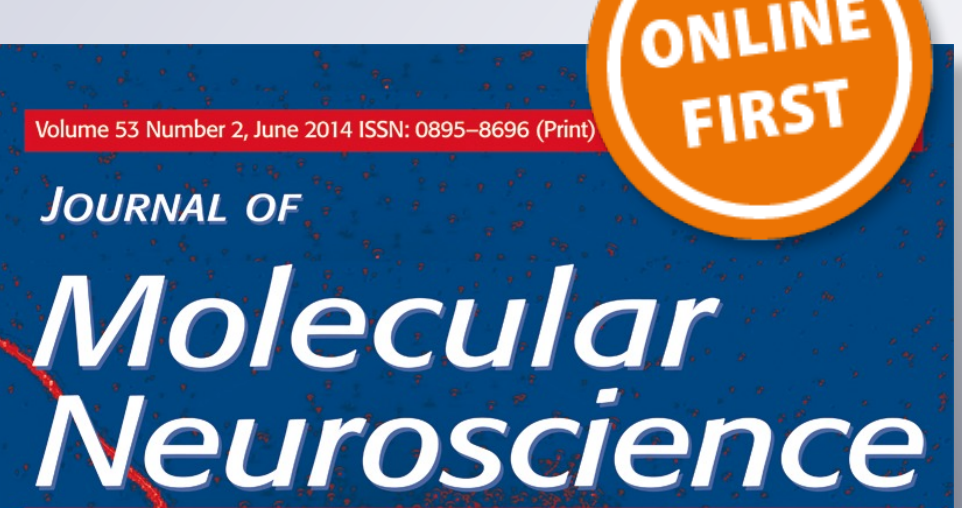

Editor-in-Chief: ILLana Gozes, PhD

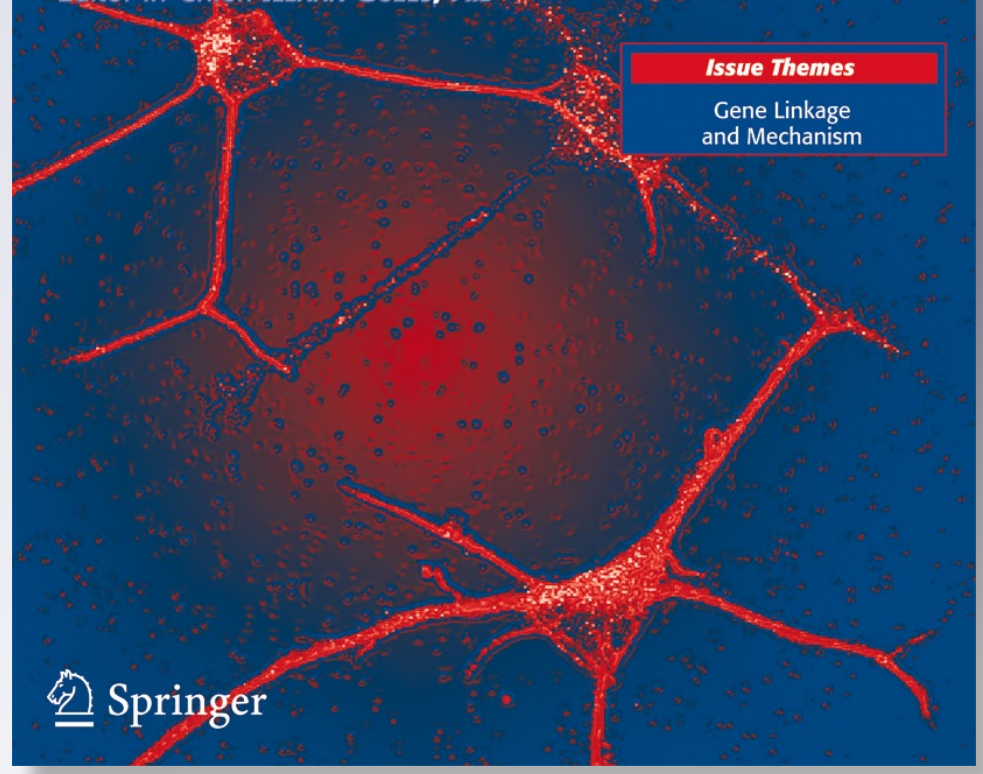

㩏 Springer 
Your article is protected by copyright and all rights are held exclusively by Springer Science +Business Media New York. This e-offprint is for personal use only and shall not be selfarchived in electronic repositories. If you wish to self-archive your article, please use the accepted manuscript version for posting on your own website. You may further deposit the accepted manuscript version in any repository, provided it is only made publicly available 12 months after official publication or later and provided acknowledgement is given to the original source of publication and a link is inserted to the published article on Springer's website. The link must be accompanied by the following text: "The final publication is available at link.springer.com". 


\title{
Differential mtDNA Damage Patterns in a Transgenic Mouse Model of Machado-Joseph Disease (MJD/SCA3)
}

\author{
Amanda Ramos • Nadiya Kazachkova • Francisca Silva • \\ Patrícia Maciel • Anabela Silva-Fernandes • \\ Sara Duarte-Silva • Cristina Santos • Manuela Lima
}

Received: 15 April 2014 / Accepted: 19 June 2014

(C) Springer Science+Business Media New York 2014

\begin{abstract}
Mitochondrial dysfunction has been associated with late onset neurodegenerative disorders, among which is Machado-Joseph disease (MJD/SCA3). In a previous study, using a transgenic mouse model of MJD, we reported a decrease in mitochondrial DNA (mtDNA) copy number and an accumulation of the 3876-bp deletion with age and with phenotype development. We extended this study by analyzing the pattern of mtDNA depletion and the accumulation of the 3876bp deletion in 12 older transgenic (TG) and 4 wild-type (wt) animals, and by investigating the accumulation of somatic mutations in the D-loop region in 76 mice (42 TG and 34 $w t)$. mtDNA damage was studied in TG and wt mice at different ages and tissues (blood, pontine nuclei, and hippocampus). Results for older mice demonstrate an accumulation of the
\end{abstract}

Electronic supplementary material The online version of this article (doi:10.1007/s12031-014-0360-1) contains supplementary material, which is available to authorized users.

A. Ramos $(\bowtie) \cdot$ N. Kazachkova $\cdot$ F. Silva $\cdot$ M. Lima

Centre of Research in Natural Resources (CIRN),

Department of Biology, University of the Azores,

Ponta Delgada, Portugal

e-mail: amanda.ramos.reche@gmail.com

A. Ramos $\cdot$ N. Kazachkova $\cdot$ F. Silva $\cdot$ M. Lima Institute for Molecular and Cell Biology (IBMC),

University of Porto, Porto, Portugal

P. Maciel $\cdot$ A. Silva-Fernandes $\cdot$ S. Duarte-Silva

Life and Health Sciences Research Institute (ICVS), School of Health

Sciences, University of Minho, Braga, Portugal

P. Maciel $\cdot$ A. Silva-Fernandes $\cdot$ S. Duarte-Silva

ICVS/3B's-PT Government Associate Laboratory,

Braga/Guimarães, Portugal

\section{Santos}

Unitat d'Antropologia Biològica, Departament (BABVE),

Universitat Autònoma de Barcelona, Cerdanyola del Vallès,

Barcelona, Spain
mtDNA 3867-bp deletion with age, which was more pronounced in TG animals. Furthermore, the tendency for mtDNA copy number decrease with age, in all analyzed tissues of TG and wt animals, was also confirmed. No point mutations were detected in the D-loop, neither in TG nor wt animals, in any of the tissues analyzed. Due to the absence of mtDNA somatic mutations, we can suggest that mtDNA point mutation accumulation cannot be used to monitor the development and progression of the phenotype in this mouse model and likely in any MJD mice model. The present results further confirm not only the association between mtDNA alterations (copy number and deletions) and age, but also between such alterations and the expression of the mutant ataxin-3 in TG mice.

Keywords Mitochondrial DNA · mtDNA damage ·

Machado-Joseph disease · Transgenic mouse model ·

Neurodegenerative disorder $\cdot$ Polyglutamine disorder

\section{Introduction}

Over the past two decades, multiple studies have provided evidence on the relevance of mitochondrial biology in neurological disorders (Martin 2010) as well as in the aging process (Larsson 2010). Mitochondrial dysfunction is being extensively studied in patients with adult onset neurodegenerative disorders such as poly-Q-related ataxias, among which is MachadoJoseph disease (MJD). MJD/SCA3 (OMIM 109150; ORPHA98757) is caused by a mutation in ataxin-3, a protein encoded by the ATXN3 gene (for a revision on MJD see Bettencourt and Lima (2011)). Although some studies have reported the presence of mitochondrial DNA (mtDNA) damage in MJD patients (Liu et al. 2008; Yu et al. 2009; Zheng et al. 2012), the way by which mitochondrial impairment and oxidative stress are actually involved in the onset and progression of the disease is not clear. A previous study from our group 
(Kazachkova et al. 2013b), using 8-, 16-, and 24-week old transgenic (TG) mice of MJD expressing the mutated ataxin-3 and displaying a motor phenotype (Silva-Fernandes et al. 2010), reported mtDNA depletion and an increase in the level of the 3867-bp deletion (the homolog of the 4977-bp deletion in humans, considered a marker of aging). Aiming to better understand the pattern of mtDNA damage in MJD, we extended this previous study by (1) analyzing the pattern of mtDNA depletion and the accumulation of the 3876-bp deletion in older TG animals and comparing them with wild-type (wt) littermates and (2) sequencing the D-loop, a mutation-prone region of mtDNA (the most variable segment in mammalian mitochondrial genomes (Attardi and Schatz 1988; Druzhyna et al. 2008)), to investigate the pattern of accumulation of somatic mutations in both TG and wt animals.

\section{Material and Methods}

Mouse Model and Experimental Design

A TG mouse model of the early stages of MJD (Mus musculus, strain C57B1/6, line CMVMJD94) developed in the Lab of P. Maciel was used in the present study (Silva-Fernandes et al. 2010). These TG mice ubiquitously express the full-length mutant human ataxin-3 and display a motor phenotype. The mouse model used also mimics some key features that are common in MJD patients, namely CAG repeat instability, neurological damage, and brain pathology (Silva-Fernandes et al. 2010). The sample selection of TG and wt mice is shown in
Table 1. Affected tissue corresponded to pontine nuclei $(\mathrm{Pn})$ and non-affected corresponded to hippocampus $(\mathrm{Hp})$ as well as blood (Bl) (Table 1). Overall, 180 samples were analyzed for early age groups ( $90 \mathrm{TG}$ and $90 \mathrm{wt}$ ) and 32 samples for late age groups (24 TG and $8 \mathrm{wt}$ ) (Table 1). A total of 76 mice were used to study the accumulation of somatic mutations (42 TG and 34 $\mathrm{wt})$; the late age groups were analyzed to study the mtDNA copy number and the 3867-bp deletion (12 TG and $4 \mathrm{wt}$ ).

The animals used in the present work were maintained in accordance with European regulations (European Union Directive 86/609/EEC). Animal facilities and the people directly involved in animal experiments were certified by the Portuguese regulatory entity-Directorate General for Veterinary Medicine. All protocols were approved by the joint Animal Ethics Committee of the Life and Health Sciences Research Institute, University of Minho and the Institute for Molecular and Cell Biology, University of Porto, Porto, Portugal.

DNA Isolation, PCR Amplification, and Sequencing

DNA was extracted using the Puregene DNA isolation kit (Gentra Systems), and the size of the CAG tract was assessed as previously described (Silva-Fernandes et al. 2010). Mitochondrial D-loop (positions 15423 to 16299) was amplified using a new designed primer pair L15349 and H133 based on the M. musculus mitochondrion complete genome reference sequence (NC_005089.1). As previously demonstrated, low heteroplasmy levels can be detected with confidence using an automated sequencing system, provided that a
Table 1 Sample selection of transgenic (TG) and wild-type (WT) mice used in the present study ( $P n$ pontine nuclei; $H p$ hippocampus)

\begin{tabular}{|c|c|c|c|c|c|c|c|}
\hline \multirow[t]{2}{*}{ Mice } & \multirow[t]{2}{*}{ Age (weeks) } & & \multirow{2}{*}{$\begin{array}{l}\text { Number of } \\
\text { mice }\end{array}$} & \multicolumn{4}{|c|}{ Number of samples } \\
\hline & & & & Pn & $\mathrm{Hp}$ & Blood & Total samples \\
\hline \multirow[t]{7}{*}{ TG } & \multirow[t]{3}{*}{ Early age group } & 8 & 10 & 10 & 10 & 10 & 30 \\
\hline & & 16 & 10 & 10 & 10 & 10 & 30 \\
\hline & & 24 & 10 & 10 & 10 & 10 & 30 \\
\hline & Total & & 30 & 30 & 30 & 30 & 90 \\
\hline & \multirow[t]{2}{*}{ Late age group } & 60 & 6 & 6 & 6 & - & 12 \\
\hline & & 72 & 6 & 6 & 6 & - & 12 \\
\hline & Total & & 12 & 12 & 12 & - & 24 \\
\hline \multicolumn{3}{|c|}{ Total number of mice } & 42 & \multicolumn{3}{|c|}{ Total number of samples } & 114 \\
\hline \multirow[t]{7}{*}{ WT } & \multirow[t]{3}{*}{ Early age group } & 8 & 10 & 10 & 10 & 10 & 30 \\
\hline & & 16 & 10 & 10 & 10 & 10 & 30 \\
\hline & & 24 & 10 & 10 & 10 & 10 & 30 \\
\hline & Total & & 30 & 30 & 30 & 30 & 90 \\
\hline & \multirow[t]{2}{*}{ Late age group } & 60 & 2 & 2 & 2 & - & 4 \\
\hline & & 72 & 2 & 2 & 2 & - & 4 \\
\hline & Total & & 4 & 4 & 4 & - & 8 \\
\hline \multicolumn{3}{|c|}{ Total number of mice } & 34 & \multicolumn{3}{|c|}{ Total number of samples } & 98 \\
\hline
\end{tabular}



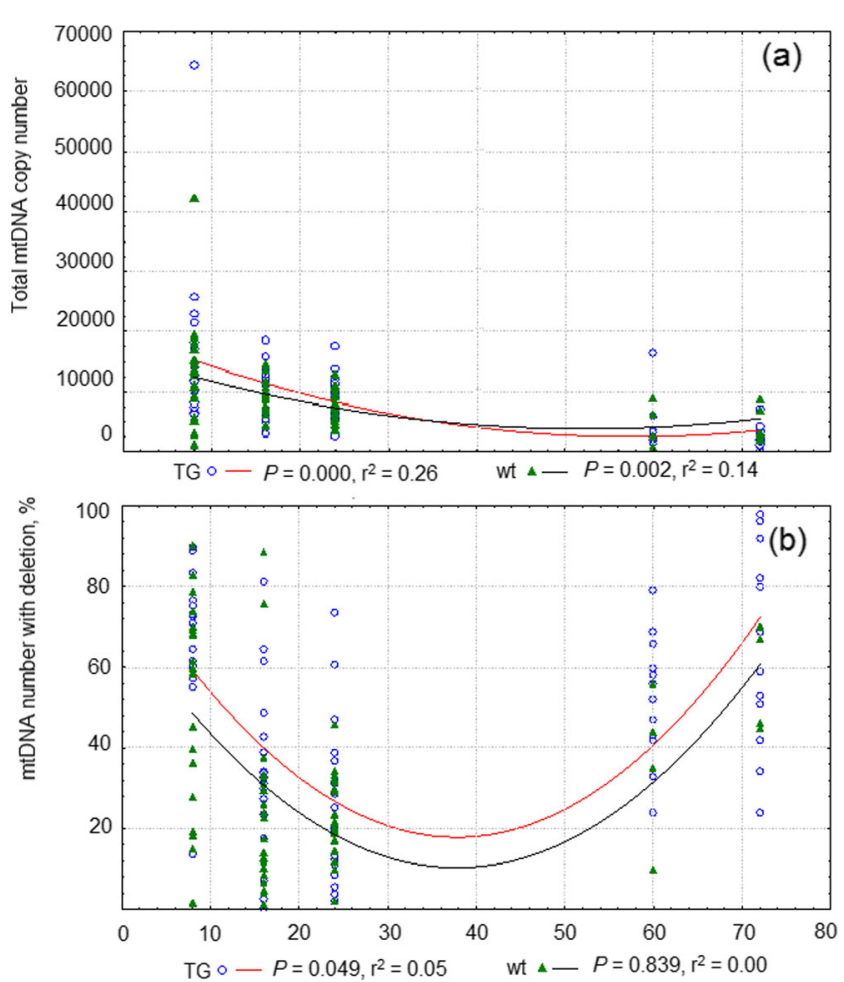

Fig. 1 Correlation of mtDNA copy number and mtDNA deletion percentage with age (weeks). The line in the graph represents a polynomial trend line. a mtDNA copy number versus age; b mtDNA deletion percentage $\%$ versus age

good sequencing strategy and an accurate procedure of heteroplasmy detection and validation are used (Ramos et al. 2013). Therefore, all samples were fully sequenced and purified using the BigDye ${ }^{\circledR}$ Terminator v3.1 Cycle Sequencing Kit (Applied Biosystems) according to the manufacturer's instructions. Sequences were run in an ABI 3130XL sequencer (Applied Biosystems) at the Servei de Genòmica, Universitat Autònoma de Barcelona. Sequences were aligned with the M. musculus mtDNA reference sequence (NC_005089.1), using SeqScape v2.5 software (Applied Biosystems). To exclude sequencing errors, heteroplasmic authentication criteria previously described by Santos et al. (2005) were applied.

\section{Quantitative Real-Time PCR}

Determination of the mtDNA copy number and quantitative detection of the 3867-bp deletion were performed by fluorescence-based quantitative real-time PCR (FQ-PCR) as described by Kazachkova et al. (2013b).

\section{Results and Discussion}

mtDNA Depletion and 3867-bp Deletion Accumulation

To obtain a more complete picture of the pattern of mtDNA damage (copy number and deletions), data published by our group (Kazachkova et al. 2013b) were extended. In our previously published work, three age groups $(8,16$, and 24 weeks) were analyzed for each tissue (Pn, Hp, and $\mathrm{Bl}$ ), in both TG and wt animals (Kazachkova et al. 2013b). In the present work, we have analyzed Pn and Hp samples from older animals, namely 60- and 72-week old TG and wt mice (Table 1). The results obtained (Fig. 1a) confirmed the global tendency for mtDNA copy number decrease with age. Interestingly, TG animals presented a significantly more evident accumulation of the 3867-bp deletion than wt (MannWhitney $U$ Test: $Z=2.74 ; p=0.0062$ ) (Fig. 1b). Although not significant, correlation between brain tissues in TG and wt animals demonstrated a more evident copy number decrease as well as a more evident accumulation of deletion in $\mathrm{Pn}$, the affected brain region (Fig. 2). The present results allow us to confirm the presence of a statistically significant accumulation of the 3867-bp deletion in TG mice; furthermore, we corroborate the tendency for mtDNA copy number decrease in TG mice previously reported by Kazachkova et al. (2013b).
Fig. 2 Correlation of mtDNA copy number (a) and mtDNA deletion percentage (b) with mtDNA origin (tissue)

\section{Mean total mtDNA copy number in} tissues

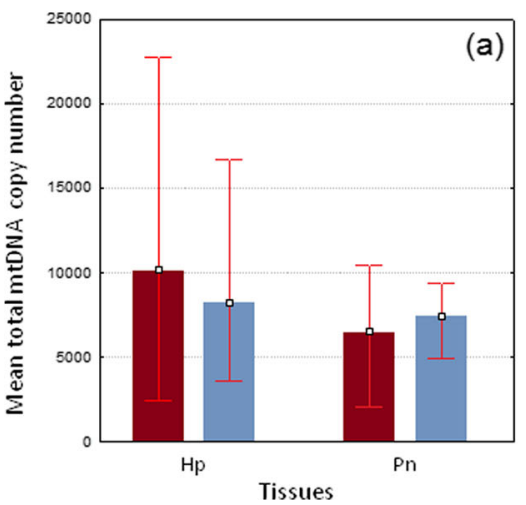

Mean mtDNA number with deletion (\%) in tissues

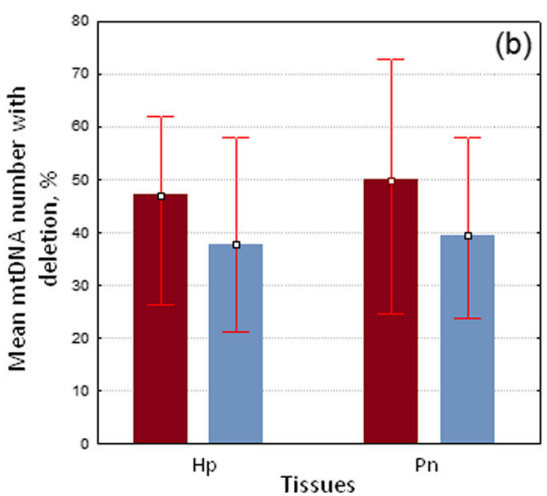


Analysis of Somatic Mutations in the Mitochondrial D-Loop

Sequence analysis of the mitochondrial D-loop of the CMVMJD94 mice and their wt littermates revealed the absence of point mutations in all samples (Supplemental material). Neither fixed mutations nor mutations in heteroplasmy were observed in any of the affected or unaffected tissues (Supplemental material). No differential pattern of mtDNA point somatic mutation accumulation was thus observed between TG and wt mice. The results obtained indicate that the murine pattern of mtDNA somatic mutation accumulation is less pronounced than in humans, a finding in accordance with most previous studies, which, with the exception of the work of Khaidakov et al. (2003), reported an absence of mtDNA point mutations in mice (Goios et al. 2007; Kazachkova et al. 2013a; Song et al. 2005; Dai et al. 2005; Ameur et al. 2011; Ferris et al. 1983). In the study of Song et al. (2005), the authors analyzed the D-loop in samples of brain, skeletal muscle, heart, and other tissues from aged mice, but were unable to find point mutations. Similar results were presented by Goios et al. (2007); these authors found no mutations in the D-loop of 32 complete mitochondrial genomes of the 16 inbred strains analyzed.

In humans, for the whole mtDNA molecule, the frequency of point heteroplasmic individuals exceeds $24 \%$ (Ramos et al. 2013). Specifically, in the D-loop, $8.2 \%$ of individuals present point heteroplasmy. Thus, in humans, one point heteroplasmy is expected in each 12 individuals. Using this expectation, the sample size needed to observe the presence of at least one heteroplasmic individual, with $95 \%$ of confidence, is 34 . In this sense, at least two heteroplasmic mice would be expected, since 76 mice have been analyzed to study the accumulation of somatic mutations (Table 1). Moreover, and given the putative negative effect of the mutant ataxin-3 on mtDNA integrity (Yu et al. 2009), higher frequencies of mtDNA point heteroplasmy in TG animals compared to wt mice would be expected. We can therefore conclude that the accumulation of point mutations in the D-loop of the mtDNA is not an indicator of mtDNA damage in the present and likely in any MJD mice model, and therefore its utility in the study of the involvement of mtDNA in MJD development is limited.

\section{Final Remarks}

Despite the differences between humans and mice, namely on what concerns physiological properties, disease pathogenesis, and life history, mouse models have been frequently used in the understanding of the process of mitochondrial alterations, mainly because they share genomic similarities. Kazachkova et al. (2013a) performed a comparative revision of studies focused on mtDNA damage (copy number alterations, accumulation of deletions, and of point mutations) carried out in humans and mice. The compilation showed consistent results among studies with a similar pattern of mtDNA deletions for humans and mice. Contradictory results, however, were reported for copy number and mtDNA point mutations accumulation (Kazachkova et al. 2013a).

Results from our study evidenced a pattern of mtDNA damage consistent with that reported by Kazachkova et al. (2013a). The presence of a statistically significant accumulation of the 3867-bp deletion was evidenced in a mouse model of MJD, being in accordance with the tendency reported in the revision of Kazachkova et al. (2013a). The absence of a significant mtDNA copy number decrease would be in line with the discrepancies observed among studies (Kazachkova et al. 2013a); thus, further studies would be necessary to elucidate this pattern. mtDNA point mutations accumulation has been clearly associated with age in humans, but not in mice (revised in Kazachkova et al. (2013a)); our study reports the lack of mtDNA somatic mutations, either at fixed or heteroplasmic level in TG mice, suggesting that mtDNA point mutation accumulation is not a useful indicator to monitor the development of the phenotype in the CMVMJD94 TG model. We can postulate that a similar pattern could be observed in mouse models of other neurodegenerative disorders, this hypothesis requiring validation.

The present results further confirm not only the association between mtDNA alterations (copy number and deletions) and age, but also between such alterations and the expression of the mutant ataxin-3 in TG mice.

Acknowledgments NK and AR are a Fundo Regional para a Ciência postdoctoral fellow (M3.1.7/F/002/2008 and M3.1.7/F/031/2011). This work was partially supported by Generalitat de Catalunya (SGR 2009-566).

Conflict of Interest The authors declare that they have no conflict of interest.

\section{References}

Ameur A, Stewart JB, Freyer C, Hagstrom E, Ingman M, Larsson NG et al (2011) Ultra-deep sequencing of mouse mitochondrial DNA: Mutational patterns and their origins. PLoS Genet 7(3):e1002028

Attardi G, Schatz G (1988) Biogenesis of mitochondria. Annu Rev Cell Biol 4:289-333

Bettencourt C, Lima M (2011) Machado-Joseph Disease: From first descriptions to new perspectives. Orphanet J Rare Dis 6

Dai JG, Min JX, Xiao YB, Lei X, Shen WH, Wei H (2005) The absence of mitochondrial DNA diversity among common laboratory inbred mouse strains. J Exp Biol 208(Pt 23):4445-4450

Druzhyna NM, Wilson GL, LeDoux SP (2008) Mitochondrial DNA repair in aging and disease. Mech Ageing Dev 129(7-8):383-390

Ferris SD, Sage RD, Prager EM, Ritte U, Wilson AC (1983) Mitochondrial DNA evolution in mice. Genetics 105(3):681-721

Goios A, Pereira L, Bogue M, Macaulay V, Amorim A (2007) mtDNA phylogeny and evolution of laboratory mouse strains. Genome Res 17(3):293-298 
Kazachkova N, Ramos A, Santos C, Lima M (2013a) Mitochondrial DNA damage patterns and aging: Revising the evidences for humans and mice. Aging Dis 4(6):337-350

Kazachkova N, Raposo M, Montiel R, Cymbron T, Bettencourt C, SilvaFernandes A et al (2013b) Patterns of mitochondrial DNA damage in blood and brain tissues of a transgenic mouse model of MachadoJoseph disease. Neurodegener Dis 11(4):206-214

Khaidakov M, Heflich RH, Manjanatha MG, Myers MB, Aidoo A (2003) Accumulation of point mutations in mitochondrial DNA of aging mice. Mutat Res 526(1-2):1-7

Larsson NG (2010) Somatic mitochondrial DNA mutations in mammalian aging. Annu Rev Biochem 79:683-706

Liu CS, Cheng WL, Kuo SJ, Li JY, Soong BW, Wei YH (2008) Depletion of mitochondrial DNA in leukocytes of patients with poly-Q diseases. J Neurol Sci 264(1-2):18-21

Martin LJ (2010) Mitochondrial and cell death mechanisms in neurodegenerative diseases. Pharm (Basel) 3(4):839-915

Ramos A, Santos C, Mateiu L, Gonzalez Mdel M, Alvarez L, Azevedo L et al (2013) Frequency and pattern of heteroplasmy in the complete human mitochondrial genome. PLoS One 8(10):e74636
Santos C, Montiel R, Sierra B, Bettencourt C, Fernandez E, Alvarez $L$ et al (2005) Understanding differences between phylogenetic and pedigree-derived mtDNA mutation rate: a model using families from the Azores Islands (Portugal). Mol Biol Evol 22(6):1490-1505

Silva-Fernandes A, Costa Mdo C, Duarte-Silva S, Oliveira P, Botelho CM, Martins L et al (2010) Motor uncoordination and neuropathology in a transgenic mouse model of Machado-Joseph disease lacking intranuclear inclusions and ataxin-3 cleavage products. Neurobiol Dis 40(1):163-176

Song X, Deng JH, Liu CJ, Bai Y (2005) Specific point mutations may not accumulate with aging in the mouse mitochondrial DNA control region. Gene 350(2): 193-199

Yu YC, Kuo CL, Cheng WL, Liu CS, Hsieh M (2009) Decreased antioxidant enzyme activity and increased mitochondrial DNA damage in cellular models of Machado-Joseph disease. J Neurosci Res 87(8):1884-1891

Zheng Y, Luo X, Zhu J, Zhang X, Zhu Y, Cheng H et al (2012) Mitochondrial DNA 4977 bp deletion is a common phenomenon in hair and increases with age. Bosn J Basic Med Sci 12(3):187-192 